\title{
Single step encapsulation process of tamoxifen in biodegradable polymer using supercritical anti-solvent (SAS) process
}

\begin{abstract}
Tamoxifen is an anti-estrogen drug which has shown tremendous excellent recovery rate in breast cancer treatment. However, after a long term of consumption, patients are prone to experience side effects of tamoxifen. These side effects however can be reduced in controlled drug delivery, using encapsulation within biodegradable method. Supercritical anti-solvent (SAS) process was chosen to synthesize tamoxifen encapsulated in poly-l-lactic using supercritical carbon dioxide as the anti-solvent. Conventionally, this encapsulation was carried out using processes such as solvent evaporation, spray drying and high pressure homogenization process. In SAS process, only a single step precipitation process was required to produce encapsulated particles and particles can be directly used upon process completion. Techniques such as Fourier transform infrared spectroscopy (FTIR), X-ray diffraction (XRD), differential scanning calorimetry (DSC), scanning electron microscopy (SEM), and energy dispersive x-ray analysis (EDX) were used to characterize the powder from SAS process and compared with raw tamoxifen as a reference. It was noted crystallinity of tamoxifen changes from crystalline to amorphous due to rapid precipitation process, but thermal properties such as melting point of tamoxifen retains after SAS process. From EDX analysis, it was found that no trace of solvent existed in the final product, thus indicating solvent was completely removed from the final product of SAS process.
\end{abstract}

Keyword: Supercritical anti-solvent; Tamoxifen; Controlled drug delivery; Encapsulation; Breast cancer; Supercritical fluid 
